smooth muscle tumour of uterine origin, occurring within the lung. Just over a hundred individual cases have been described in the literature; with two series of ten patients each, from continental Europe and North America.

Objectives We sought to present the first comprehensive descriptive series from a contemporary U.K. population.

Methodology Patients at a single U.K. thoracic surgical centre, between 2003 and 2017, were identified from prospective histology databases. Retrospective data was collected from physical and electronic data sources, and cross-referenced for accuracy.

Results 6 patients - all postmenopausal females - were identified over a 15 year period. Average age was $44 \pm 8$ years (mean $\pm \mathrm{SD}$ ). Half of the patients were asymptomatic with an incidental finding of pulmonary nodules, whilst the remaining $50 \%$ complained of nonspecific respiratory symptomatology. Plain imaging of the chest failed to reveal any abnormality in $2(33.3 \%)$ patients. $5(88.3 \%)$ patients had multiple lesions median of 9 (range 2 to 12) - with bilateral distribution; measuring a median $11 \mathrm{~mm}$ (range 7 to 27) in size on crosssectional imaging. All patients underwent diagnostic surgical wedge biopsy, with $5(88.3 \%)$ procedures completed thoracoscopically; and no perioperative morbidity. 4 (66.7\%) patients had a history of previous hysterectomy, and a further patient underwent a hysterectomy following the diagnosis of BML all for uterine leiomyomata. $4(66.7 \%)$ patients underwent oophorectomy, whilst one patient required hormonal suppression therapy. Survival was $100 \%$ at a median follow up of 37 months (range 4 to 150 ).

Conclusion BML is a rare clinical entity accounting for a small proportion of patients presenting with pulmonary nodules. Following successful tissue diagnosis, outcomes with conservative or medical management are excellent.

\section{P263 DAILY "VIRTUAL" CANCER CLINIC- THE END OF THE ONE STOP CLINIC?}

DA Tarpey, K Hughes, AG Wight. Wirral University Teaching Hospital NHS Foundation Trust, Wirral, Merseyside, UK
Cancer investigation targets are set nationally with current 14 day referral to review and 62 days to treatment. Nationally and locally there has been difficulty meeting these current standards and timescales are being revised with published NHS strategy 2015-20 (28 days referral-treatment plan). Wirral University Hospital is a large non-tertiary trust who diagnosed a total of 341 lung cancers in 2015, 147 following GP urgent referrals (total 547 referrals) Previously we ran a traditional "one stop" weekly clinic with CT scan, review and same day endoscopy. Study of delayed pathways highlighted problems- lack of same day "best test", delay of arranging PET/physiology testing and anticoagulation/sedation issues were preventing same day investigations. In September 2015 we changed to a daily virtual review clinic post CT (day 5-7). First patient contact is via telephone call from CNS to explain further investigations and give contact details. We audited 3 months diagnoses following primary care referrals Sept-Nov 2015 vs 2014 and completed patient satisfaction questionnaire post diagnosis on communication and perceived management. Results- Diagnoses - 38 (2014)/36 (2015). 2015 showed reduction in average investigations (1.4 vs 1.18); outpatient attendances (2.4 vs 1.75); time to PET-CT (19.8 vs 15.7 days) and total radiological diagnoses ( 7 vs 3 ). There was no reduction in median time to diagnosis ( 20 vs 21 days) but less variance in pathways with shorter range. On survey $16 / 18$ of patients rated the care/communication as excellent. For the 36 new diagnoses post implementation we saved equivalent of 23 follow-up appointments and 9 invasive investigations (equating $£ 30,000$ /year savings) within the group diagnosed with cancer. We feel the change from traditional one-stop outpatient clinics to a more individual case based management with virtual review and non-OPD based communication is essential to develop lung cancer pathways and would advise other units to adopt similar

\section{P264 TELEPHONE CONSULTATIONS FOR PATIENTS WITH NEWLY DIAGNOSED LOW RISK LUNG NODULES}

MPT Kennedy, KA Rodger, JM Robson, E Paramasivam, MEJ Callister. Leeds Teaching Hospitals NHS Trust, Leeds, UK

10.1136/thoraxjn|-2017-210983.406

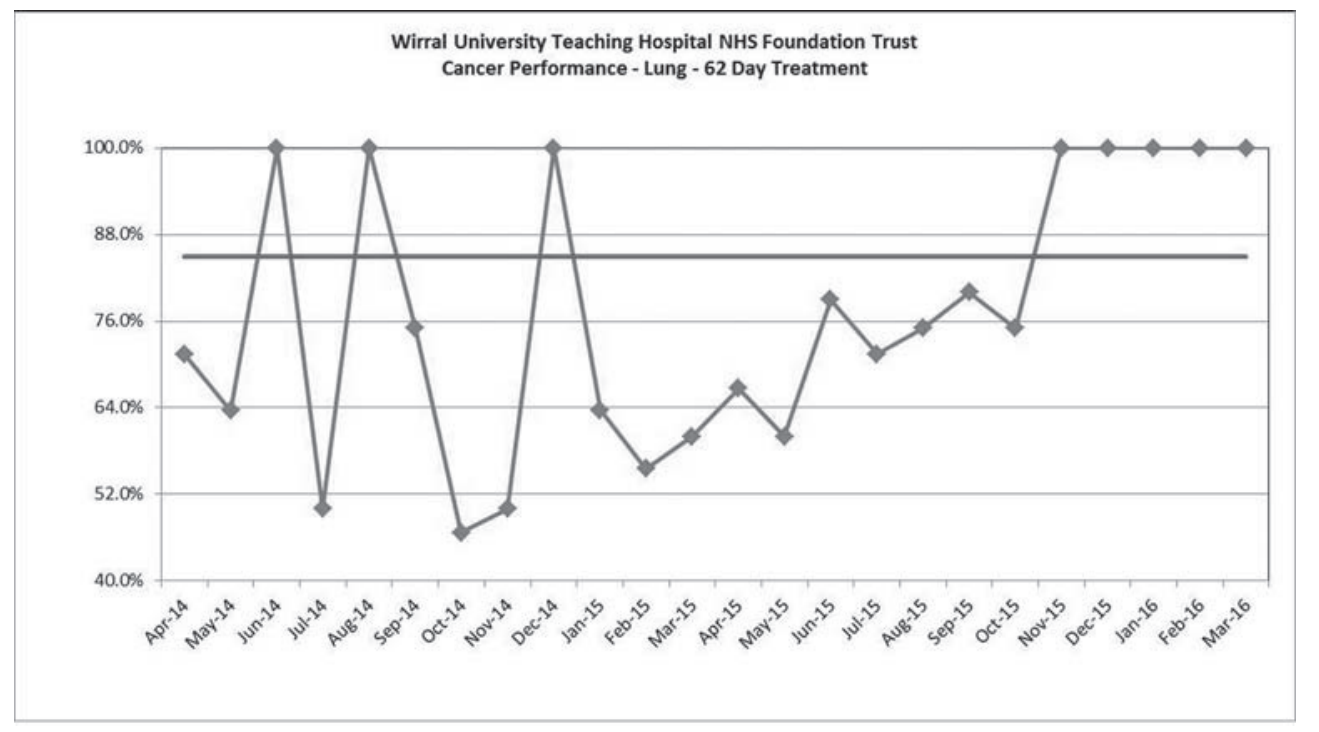


Introduction Lung nodules are a common incidental finding on chest imaging. Their identification on CXR or CT thorax is a common trigger for referral to the lung cancer MDT. Low risk nodules $\left(<8 \mathrm{~mm}\right.$ diameter, $<300 \mathrm{~mm}^{3}$ volume, or $<10 \%$ risk of malignancy on Brock Model) do not require urgent intervention, but may require CT surveillance. These patients, however, are usually aware that they have been referred as "suspected cancer" and require prompt reassurance. In Leeds Teaching Hospitals, patients with low risk nodules were previously brought to lung cancer fast-track clinics for initial consultation. In 2016, we introduced $10 \mathrm{~min}$ telephone appointments for patients with new lung nodules, followed by a letter and an information leaflet to the patient. The aim is to improve access to fast-track appointments for patients with a CT scan showing suspected cancer, while allowing patients with low risk lung nodules to receive reassurance sooner and with less inconvenience. We aim to assess the impact of this service on access to fast-track clinics and patient experience.

Methods Patients with new low risk pulmonary nodules were identified from MDT records. We sent surveys to the most recent 24 patients that attended fast-track clinics and 24 patients that had received telephone appointments.

Results During the first three months of the Leeds Pulmonary Nodule Service, a mean of 6 patients per week received telephone consultations, projecting to 325 patients per year. This improved capacity in the fast-track clinic by 4 new patient appointments per week, equivalent to 200 per year. The survey response rate was $13(54.1 \%)$ from fast-track patients and $14(58.3 \%)$ from telephone patients; Table 1.

Discussion The Leeds Pulmonary Nodule Service has led to increased availability of fast-track appointments for patients with suspected lung cancer and improved patient satisfaction and patient-rated quality of care for patients with low risk lung nodules. The new service is currently delivered solely by consultants and this may have impacted on the survey results.

\begin{tabular}{|c|c|c|}
\hline & Type of clinic appointment & \\
\hline & Face-to-face & Telephone \\
\hline \multirow[t]{2}{*}{ Travel time } & Mean 60 min each way & $\mathrm{N} / \mathrm{A}$ \\
\hline & Two patients $>2$ hours each way & \\
\hline Appointment on time & $46.2 \%$ & $85.7 \%$ \\
\hline \multirow[t]{2}{*}{ Rating of explanation } & Mean $4.1 / 5$ & Mean $4.6 / 5$ \\
\hline & $69.2 \%$ score $4-5 / 5$ & $85.7 \%$ score $4-5 / 5$ \\
\hline Opportunity for questions & $84.6 \%$ & $85.7 \%$ \\
\hline Provided written information & $61.5 \%$ & $92.9 \%$ \\
\hline \multirow[t]{2}{*}{ Overall rating of care } & Mean $4.1 / 5$ & Mean $4.6 / 5$ \\
\hline & $72.8 \%$ score $4-5 / 5$ & $92.8 \%$ score $4-5 / 5$ \\
\hline \multicolumn{3}{|c|}{ How would patients prefer to have had their appointment? } \\
\hline Face-to-face clinic & $66.7 \%$ & $14.3 \%$ \\
\hline No preference & $8.3 \%$ & $28.6 \%$ \\
\hline Telephone clinic & $25.0 \%$ & $57.1 \%$ \\
\hline
\end{tabular}

\section{P265 SURVEY OF NORTH WEST ENGLAND CT FOLLOW-UP OF PATIENTS POST RADICAL TREATMENT FOR LUNG CANCER}

P Griffiths, DG Fullerton, DC Lees. Mid Cheshire NHS Foundation Trust, Crewe, UK
Introduction There is not currently consensus in the UK regarding optimal follow up of patients with non-small cell lung cancer (NSCLC) after radical treatment. Survivorship clinics aim to detect treatment related complications, assess for disease recurrence, support patients (and families) as well as detect new primaries. Computed tomography (CT) is superior to chest x-ray (CXR) for NSCLC follow-up and although several professional bodies have issued guidance on CT follow-up after treatment, real-world practice on when to perform CT follow-up varies according to local protocols, resources as well as patient-related variables.

Aim To determine local patterns of CT surveillance in patients treated radically for NSCLC.

Methods An online questionnaire was sent to 40 different specialists involved in lung cancer treatment in the North West of England. They included respiratory physicians, thoracic surgeons, and clinical and medical oncologists.

Results 21 questionnaires were completed. Surveillance patterns varied between the treatment modality delivered and specialty. Following curative surgery $(n=19$ respondents): Short-term CT surveillance intervals varied between no routine CT $(n=5), 3-$ 6 monthly $(n=4), 1-2$ yearly $(n=10)$. Following radical radiotherapy $(n=16)$ : Frequency of CT varied between no routine CT $(n=4)$, 3-6 monthly $(n=5)$ and $1-2$ yearly $(n=7)$. Following Stereotactic Ablative Radiotherapy (SABR) $(n=7)$ : Frequency of CT surveillance varied from 3-6 monthly $(n=5)$ to $1-2$ yearly $(n=2)$. The total duration of routine follow-up also varied from 'Indefinitely' $(n=2)$ to 5 years $(n=16)$, and 10 years $(n=3)$.

Conclusion This survey has demonstrated that wide variation exists in the NW England in relation to when to perform CT scans in patients who have had a radical treatment for NSCLC. There is no standardised follow up protocol for this patient group in NW England. An agreed protocol for follow up of patients after radical treatment for NSCLC based on variables that predict recurrence is needed. It is hoped that data from on-going research should help to inform follow up protocols in the future. We have demonstrated that there is a need for a more uniform and evidence based strategy for CT scan follow up of patients with NSCLC.

\section{P266 EARLY LUNG CANCER TEAM INTERVENTION IN EMERGENCY ADMISSIONS}

J Dunbar, M Walshaw, M Ledson, A Mclver, J Hughes, N Maddock, C Smyth. The Royal Liverpool University Hospital NHS Trust, Liverpool, UK

\subsection{6/thoraxjnl-2017-210983.408}

Introduction Although the majority of cases of suspected lung cancer can be managed as outpatients where the diagnostic pathway is well developed, a proportion still present unwell via the emergency department where their subsequent journey is less certain. Along with same-day reporting for emergency scans, we have developed a rapid review service for such individuals and were interested in assessing its performance.

Methods We looked at all patients with suspected lung cancer who presented through our emergency department for the first 6 months of 2017, focussing on outcome, time to be seen following admission, and the histological diagnosis rate.

Results Of the 285 referrals to our lung cancer unit, 85 (32\%) presented through the emergency department: 21 $(25 \%)$ of these were discharged to outpatient cancer 